\title{
Playing with Systems: Tactile Games as System Prototypes
}

\begin{abstract}
The world's most pressing challenges are systemic. Our ability to survive as a species is now linked to how quickly and effectively we are able to address systemic challenges like climate change. The practice of low-resolution prototyping helps designers quickly explore and learn about ideas, but it is rarely utilized when designing for complex systems. At the same time, physical games have often been utilized in service of learning about complex systems, but they have not been used as prototypes for systemic interventions. This short paper and demo provide examples of two games that show how designers can use games as low-resolution prototypes for complex systems. These physical games will transform systems from intimidating, impenetrable, and abstract concepts, to entities that can be explored, engaged, and influenced.
\end{abstract}

\section{Keywords}

Climate Change, Prototyping Systems, Design, Games, Play

\section{Introduction}

Climate change is a systemic challenge that poses an unprecedented threat to life as we know it. Responses to climate change will require the deployment of an unprecedented breadth of initiatives at a global scale in little over a decade.[1] In order to be successful, we must be able to rapidly explore ambitious ideas as well as the risks they entail. Low-resolution prototypes are well suited to this task and although they are widely used at the product and service scale, they have yet to be employed in the design of systems. This paper demonstrates the ways physical games can be used as a low-resolution prototypes of a systems-level interventions and show how employing this type of practice can help experts and non-experts engage with complex systems in a tangible way.

\section{Complex Systems}

While complex systems are varied in form and scale, for the purposes of this paper they will be categorized by the work of Yaneer Bar-Yam. According to BarYam, these types of systems share the common traits of a diverse range of functions, interconnections, interwoven elements that each relate, and influence one another.[2] Using this framework, the challenge of climate change is one of our best examples of a complex system. Its causes are layered and inter-related and any meaningful responses to it will require shifts in the way multiple systems (such as national, regional and local governments) behave. 


\section{Prototypes as Tools for Learning}

This work builds upon the notion of prototyping from a design thinking perspective. In this domain, the practice of prototyping can be described as the rapid creation of artifacts to learn about a solution space. Prototyping is widely understood in the product and service design realm as a way to explore, gain understanding, and reduce risk.[3-7] Within this practice, prototypes are broken into low, medium and high fidelity. Lower fidelity, or low-resolution (low-res) prototypes are much faster to build and are easier to make, but they bear little resemblance to the final version of an idea

[8] When creating low-resolution prototypes, designers focus their efforts on building only what is needed to elicit feedback about critical areas of the design from their users. In this way, low-res prototypes allow designers to quickly learn and iterate towards more refined solutions.

\section{Educational Games}

Although the use of games in service of learning is not new, the work of Dieleman and Huisingh (2006) is instructive for the discussion of prototyping because they highlight the connections between games and experiential learning.[9] Some of the benefits Dieleman and Huisingh attribute to playing games, specifically the focus on experimentation and failing without real consequences, could easily be used to describe the benefits of low-res prototyping.

Learning games and prototypes do share many common traits, but there is an important distinction between the two. Educational games use play in service of a learning objective. While games-as-prototypes certainly offer some educational benefit to the player, their primary purpose is to help the design team understand as much as possible about the design space in the least amount of time.

\subsection{Examples of Low-Resolution Systems Prototypes}

In the following games, a complex system is translated into a physical experiences and objects that help a variety of stakeholders engage with these systems in a more meaningful way. These games reflect a humility that acknowledges the limitations of our ability to fully understand or account for all of the potential forces and variables within a system. Like any low-resolution prototype, these games do not attempt to replicate the full complexity of a system. Instead, the games are designed with just enough context and game mechanics to spark visceral experiences of system level changes. The simple quality of the game serves multiple purposes. It allows the games to be played by a wide range of users and it minimizes the effort required to create the prototype/game itself. If the game becomes too complicated, the focus will shift from the larger goal and intervention to the prototype.

"Earth Systems and Modern Convenience" serves as a prototype for a hypothetical initiative intended to promote sustainable practices. It utilizes the relationship between gravity and distance from the floor to simulate the ways our planet's natural and human-systems become more fragile as our population grows. The game, grows more challenging at the later phases. As a prototype, the game will be successful if it can help the designers gain greater confidence in a proposed the direction before committing any more time or money than is necessary.

The game has two key parts, a wooden disc balancing on a thin column and a series of small wooden blocks. The 24" wooden disc represents the Earth. The height of the column on which it rests corresponds to the Earth's historical (or projected) populations. The wooden blocks each represent mundane elements of modern life such as owning a car, eating non-local food, or utilizing same-day delivery services. The object of the game is to place as many blocks onto the disc without causing it to topple. Through the course of the game, the players are forced to make difficult decisions which reveal a variety of strategies, connections and intentionality about choices that are often unnoticed in daily life. The tactile nature of the game leverages existing systems, (gravity, the player's nervous system and the uncertainty of the immediate environments) which all become inputs for the game. As an example, a heavy footed pedestrian could throw off a precariously balanced disc.

If the debrief is a critical part of an educational game, as noted by Dieleman and Huisingh, it is absolutely essential for a prototype.[10] The debrief offers the 
designer a chance to understand more about the player's choices and experiences during the game. The phase of the design process, commonly referred to as "testing", helps designers understand more about their users and their proposed solution. At the Stanford University d.school, the process of testing is often referred to as "empathy with a prop".[11] The debrief after a game serves a similar purpose and the insights gained in this phase help inform the next iteration of a solution.

During the debrief of the game, it is critical that the designer remains open to the variety of discussions that emerge, even if they challenge the premise of the game. For example, in "Earth Systems and Modern Convenience," players may rightfully question why all the blocks are weighted the same when the effects of single day delivery and non-local food may have very different impacts. In remaining open to these kinds of discussions, the designers are able to get a better understanding of the ways which the players view the system and this creates space for the players to become design collaborators as well.

"Critical Responses to Climate Change" comes out of the OneClimate initiative at University of California, Davis which is spearheaded by Dr. Benjamin Houlton, Director and the author who is on the leadership team for the initiative. In order to break down the complexity of climate change into an actionable (although highly ambitious) plan for our species' survival, we distilled the challenge into three key activities. As a planet, we must remove carbon from the atmosphere, change human behavior to adopt carbon neutral lifestyles, and do all of this while adapting to the effects of climate change that are already underway. In order to share this approach with a wide audience, the author developed a metaphor in which the Earth is a leaking boat. To survive we need to bail the boat and fix the leak in the growing turbulence caused by climate change. An animated video describing the approach can be seen here. (https:// vimeo.com/270732552)

This game translates the metaphor of a sinking boat into a tangible format that is suitable for elementary school and above (Fig. 1). The leaks in the boat represent carbon accumulation in the atmosphere. The boat will sink unless the players are able to bail the boat (pulling $\mathrm{CO} 2$ out of the atmosphere), fix the leak (changing human behavior in order to adopt carbon neutral practices) and shore up the boat (responses to the effects of climate change that are already underway). This game translates some of the climate jargon and abstract concepts into physical experiences that help players experience climate change in a visceral way.

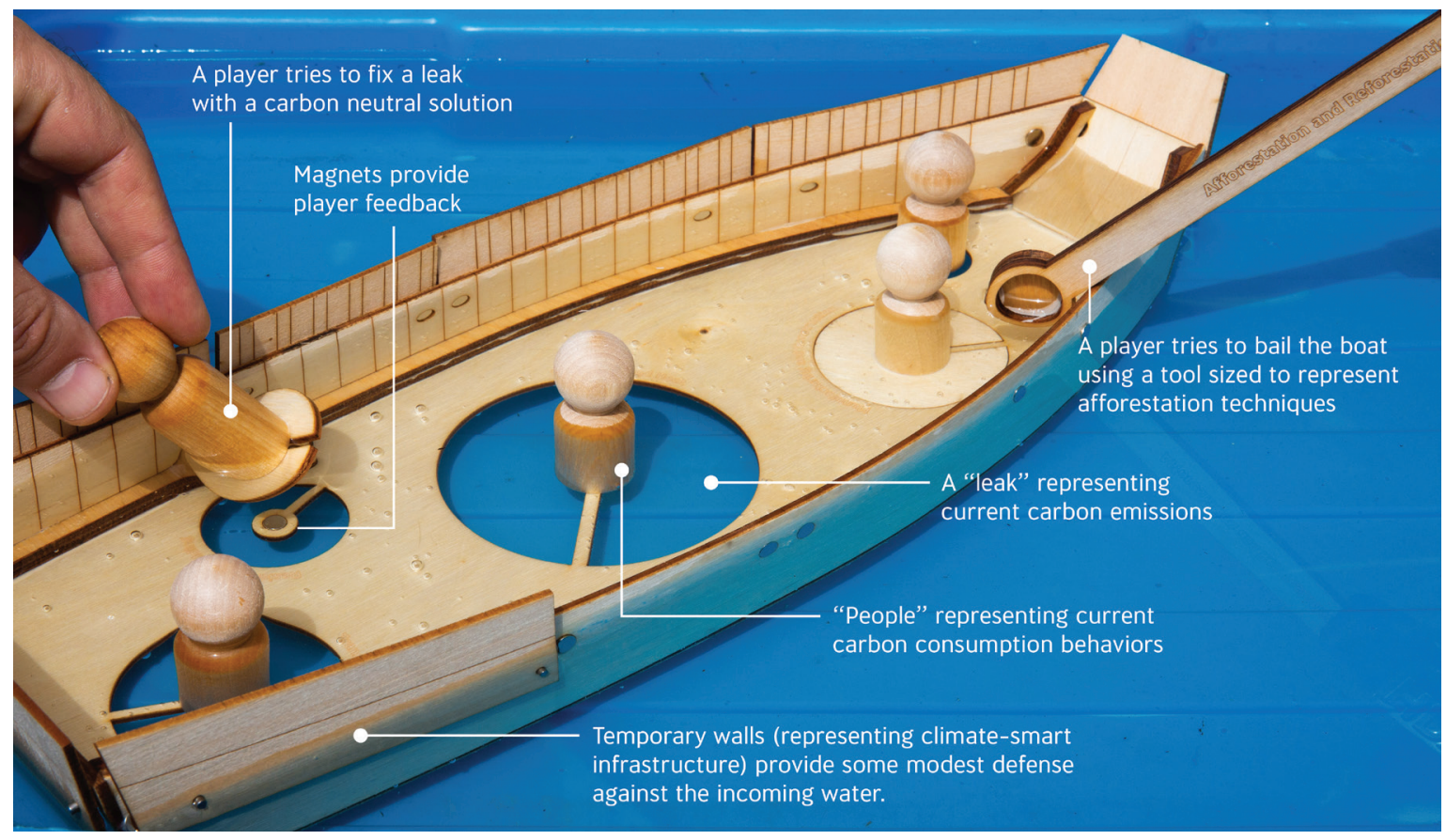

Fig. 1. An image of the game in action with key elements of the prototype called out 
Players may work as an individual or in teams. They are not allowed to touch the boat and they have a small amount of time (six minutes) to keep the boat afloat. The leaks in the boat correspond to the proportions of carbon emissions and the bailers are sized to represent the latest figures on carbon sequestration. As with Earth Systems and Modern Convenience, the efficacy this game is deeply tied to the debrief and facilitation after the game play. In the game, the role of water, magnets and collaborative efforts between players all become rich sources of insight.

\section{Conclusions}

In this paper, the author provides a theoretical framework for the ways which games can be used as prototypes for interventions in complex systems. These low-resolution prototypes can provide insight to both players and game designers which provides the ability for designers to gain greater insight into potential system interventions in less time and with less risk. The games themselves have been designed and prototyped but more follow-up research is needed to explore the potential and shortcomings to this approach. As tools for learning they are not intended to replace the full complexity of a system, but instead offer playful ways of quickly eliciting information about the dynamics, relationships and unexpected influences in the system for both player and designer.

Acknowledgements. The author wishes to thank the John Muir Institute of the Environment at UC Davis for supporting this work and Sophie Zagerman for her research assistance.

\section{References}

1. The Special Report on Global Warming of $1.5^{\circ} \mathrm{C}$ (SR15), (2018, October 8) Intergovernmental Panel on Climate Change (IPCC) Retrieved March 12, 2019, from https:// www.ipcc.ch/sr15/

2. Bar-Yam,Y. (1997) Dynamics of complex systems ( $p 1$ ) Addison-Wesley

3. Gengnagel, C., Nagy, E., and Stark, R. (2016) Rethink! Prototyping: Transdisciplinary Concepts of Prototyping (p4) Springer International

4. Brown, T., and Katz, B. (2011) "Change by Design." Journal of
Product Innovation Management $28.3: 381-83$

5. McElroy, K. (2017) Prototyping for Designers : Designing the Best Digital and Physical Products ( $X I X)$ O'Reilly

6. Kolko, J. (2017) Creative Clarity (p89) Brown Bear Publishing

7. Martin, R. (2014) Strategy is Iterative Prototyping, Harvard Business Review, June 2014 https://hbr.org/2014/06/strategyis-iterative-prototyping Retrieved March 12, 2019

8. McElroy, K. (2017) Prototyping for Designers: Designing the Best Digital and Physical Products (p40) O'Reilly

9. Dieleman, and Huisingh. "Games by Which to Learn and Teach about Sustainable Development: Exploring the Relevance of Games and Experiential Learning for Sustainability." Journal of Cleaner Production 14.9 (2006): 837-47. Web.

10. Ibid. p842-843

11. Author's personal experience over the last 10 years as a coach for the Hasso Plattner Institute of Design at Stanford University's (d.school) Design Thinking Bootcamp. The phrase "Empathy with a prop" is often referenced by the Executive Education Leadership Team of Perry Klebahn, Jeremy Utley, and Kathryn Segovia. 